\title{
Mudanças no Padrão Temporal da EMG de Músculos do Tornozelo e Pé Pré e Pós- Aterrissagem em Jogadores de Voleibol com Instabilidade Funcional
}

\author{
Changes in the EMG Temporal Pattern of Pre and Post-landing of Ankle \\ and Foot Muscles in Volleyball Players With Functional Instability
}

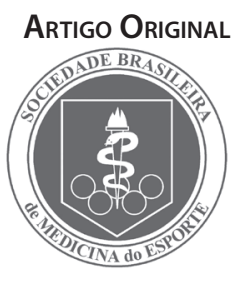

Eneida Yuri Suda', 2,3

Anita Lopes Cantuária

Isabel de Camargo Neves Sacco

1. Laboratório de Biomecânica do Movimento e Postura Humana - Departamento de Fisioterapia, Fonoaudiologia e Terapia Ocupacional, Faculdade de Medicina da Universidade de São Paulo - São Paulo, SP. 2. Centro Universitário Capital, Curso de Fisioterapia - São Paulo, SP. 3. Universidade do Grande ABC Curso de Fisioterapia -

Santo André, SP.

Endereço para correspondência: Rua Masuzo Naniwa, 105 - Bloco Tarragona - apto. 84 - Nova Mogilar 08773-535 - Mogi das Cruzes, SP. E-mail: yurisuda@usp.br

Submetido em 26/04/2007 Versão final recebida em 30/11/2007 Aceito em 28/01/2008

\section{RESUMO}

Introdução: A instabilidade funcional (IF) é a complicação mais comum após o entorse de tornozelo; acomete até 52\% dos atletas com história de entorse. O entorse de tornozelo é uma das lesões esportivas mais comuns, inclusive no voleibol, em que $90 \%$ dos entorses de tornozelo ocorrem durante a aterrissagem, após o movimento de bloqueio. A IF é definida por queixas de falseios e entorses recorrentes, sem que haja evidências clínicas de lesão ligamentar, sendo extremamente prejudicial para a prática do voleibol, pois interfere na realização dos fundamentos envolvidos na modalidade. Objetivos: Comparar os padrões temporais e de magnitude da atividade eletromiográfica dos músculos tibial anterior (TA), fibular longo (FL) e gastrocnêmio lateral (GL) durante a aterrissagem do salto vertical após a execução da habilidade do bloqueio do voleibol entre jogadores com e sem IF de tornozelo. Métodos: Foi adquirida a atividade EMG do tibial anterior, do fibular longo e do gastrocnêmio lateral em 21 atletas com IF (GI) e em 19 atletas controle (GC) - idade média de $20 \pm 4$ anos. Os envoltórios lineares foram calculados para cada um dos grupos no período de tempo entre $200 \mathrm{~ms}$ antes e $200 \mathrm{~ms}$ após o instante do impacto, determinados por meio da componente vertical da FRS. A magnitude e o instante do pico máximo de cada um dos músculos também foram determinados matematicamente. Os grupos foram comparados por meio do teste $t(\alpha=0,05)$. Resultados: 0 grupo com instabilidade apresentou instante de pico do TA mais tardio $\pm(G C=-107,4 \pm 29,6 \mathrm{~ms} ; \mathrm{Gl}=-134,0$ $\pm 26,0 \mathrm{~ms})$ e FL (GC $=-11,0 \pm 55,9 \mathrm{~ms} ; \mathrm{Gl}=-41,7 \pm 49,8 \mathrm{~ms}$ ) e menor pico de TA (GC $=68,5 \pm 17,2 \% ; \mathrm{Gl}=81,2$ $\pm 28,8 \%)$ e $F L(G C=72,9 \pm 27,3 \% ; G l=59,1 \pm 16,0 \%)$, Conclusões: Os resultados mostram um padrão de alteração mais tardio e com menor magnitude nos músculos de atletas com IF que podem predispô-los à condição de instabilidade, mesmo na ausência de lesão anatômica.

Palavras-chave: instabilidade articular, atividade motora, eletromiografia, esportes.

\section{ABSTRACT}

Introduction: The ankle sprain is one of the most common injuries in athletes, including volleyball. 90\% of ankle injuries in volleyball occur during landing after a blocking maneuver. The most common complication following ankle sprains is functional instability (FI), a condition that affect about $52 \%$ of the patients that suffered an ankle sprains. Functional ankle instability (FI) has been defined as a tendency for the foot to give way after an ankle sprain with no evidence of ligament injury. Hence, Fl is an impairing condition for volleyball performance since it interferes in its basic skills. Aims: The purpose of this study was to compare the EMG activation patterns of tibialis anterior (TA), peroneus longus (PL) and gastrocnemius lateralis (GL) in volleyball players with and without Fl during landing after the blocking movement. Methods: EMG activity was acquired for 21 subjects (mean age $20 \pm 4$ yrs) with FI (IG) and 19 control ones (CG). Linear envelopes were calculated for both groups for the time period between 200 ms before and 200 ms after the instant of impact, and time and magnitude of peak occurrence were extracted from the envelopes. Groups were compared using T test $(\alpha<0.05)$. Results: IG subjects showed a later peak occurrence for TA (CG $=-107.4$ $\pm 29.6 \mathrm{~ms} ; \mathrm{IG}=-134.0 \pm 26.0 \mathrm{~ms})$ and $\mathrm{PL}(\mathrm{CG}=-11.0 \pm 55.9 \mathrm{~ms} ; \mathrm{IG}=-41.7 \pm 49.8 \mathrm{~ms})$ and a lower peak magnitude for TA (CG = 68.5 \pm 17.2\%; FIG $=81.2 \pm 28.8 \%)$ and PL (CG = 72.9 $\pm 27.3 \%$; FIG $=59.1 \pm 16.0 \%$ ). Conclusions: These results suggest that individuals with FI present a later and lower activation pattern of muscular activity and different activation magnitudes that predispose them to ankle sprains, even in the absence of an anatomical damage.

Keywords: joint instability, motor activity, electromyography, sports. 


\section{INTRODUÇÃO}

Uma das lesões esportivas mais comuns é o entorse de tornozelo ${ }^{(1-2)}$, sendo essa, da mesma forma, a lesão aguda mais prevalente entre os praticantes de voleibol ${ }^{(3-7)}$. No voleibol, 63\% das lesões em membros inferiores são associadas às habilidades motoras de ataque e bloqueio(8) e o desempenho efetivo desse movimento depende do controle motor e da habilidade desse sistema para modificar os detalhes da ação de acordo com as circunstâncias necessárias ${ }^{(9)}$. Geralmente, essas habilidades resultam em inversão do tornozelo, causando lesão nos ligamentos laterais quando aterrissam no solo após o salto vertical(3,10). Alguns autores têm descrito que, independentemente do tratamento inicial, de 10 a 40\% dos indivíduos com história de entorses no tornozelo relatam sintomas persistentes ou reincidência das lesões, caracterizando um quadro de instabilidade crônica(2,11-13).

A instabilidade crônica do tornozelo pode ser funcional, que é definida como um quadro formado por um conjunto de sintomas tardios, que se desenvolvem após a resolução da lesão ligamentar inicial, ou seja, a queixa é presente sem que haja evidência de lesão do complexo ligamentar ${ }^{(14-19)}$. Os indivíduos com instabilidade funcional costumam apresentar queixas de entorses de recorrência na execução de atividades esportivas ou durante o simples ato de andar ${ }^{(14,20-24)}$, falseios ${ }^{(17,20-25)}$, dificuldade de andar ou correr em superfícies irregulares ${ }^{(21,24)}$ e dificuldade de realizar saltos e mudanças de direção durante a prática de atividades esportivas ${ }^{(21)}$.

O aumento do tempo de reação dos músculos fibulares ${ }^{(23-24)} \mathrm{e}$ a fraqueza dos mesmos(22) têm sido considerados como causas de instabilidade, já que atuam ativamente quando há o contato do pé com o solo durante as habilidades de locomoção, evitando eversões excessivas ${ }^{(19)}$. Alterações no tempo de reação do músculo tibial anterior em situações em que um mecanismo inversor é gerado no complexo articular do tornozelo-pé também já foram descritas ${ }^{(24-25)}$.

A maioria dos estudos nesta temática investigaram as respostas musculares em indivíduos com e sem instabilidade de tornozelo em situações extremamente controladas e/ou estáticas de simulação do mecanismo de lesão, ou seja, em situações em que há uma inversão súbita do pé(24,26), mas poucos estudos enfocaram situações ecologicamente válidas, presentes na prática esportiva, onde de fato as lesões do tornozelo ocorrem(27), tal como propõe o presente estudo.

O objetivo do presente trabalho foi comparar os padrões temporais e de magnitude da atividade eletromiográfica dos músculos tibial anterior, fibular longo e gastrocnêmio lateral entre jogadores com e sem instabilidade funcional de tornozelo. Essa comparação foi realizada durante a aterrissagem do salto vertical após a execução da habilidade do bloqueio do voleibol. Duas hipóteses são levantadas: (1) os músculos tibial anterior, fibular longo e gastrocnêmio lateral apresentam menor ativação nos indivíduos com instabilidade funcional; (2) os músculos tibial anterior, fibular longo e gastrocnêmio lateral apresentam um pico de ativação atrasada em relação ao instante da aterrissagem no bloqueio. O reconhecimento desses padrões alterados permitir-nos-á identificar a origem de uma das possíveis causas da instabilidade funcional em uma população de atletas. Esse reconhecimento é de suma importância, uma vez que pode ajudar a elucidar a causa do problema, delineando uma linha de tratamento para indivíduos que sofreram um entorse de tornozelo e, assim, prevenir o desenvolvimento da instabilidade, ou no tratamento de indivíduos que já apresentam instabilidade, diminuindo esta incapacidade no atleta.

\section{MÉTODOS}

Quarenta jogadores de voleibol profissionais de ambos os sexos com tempo de prática médio de 7,6 \pm 3,9 anos e idade média de \pm 20 \pm 4 anos foram avaliados e assinaram um termo de consentimento livre e esclarecido aprovado pelo Comitê de Ética da instituição local.

O grupo controle (GC) foi constituído de 19 sujeitos (18 do sexo masculino e um do feminino, massa média de 79,4 \pm 9,9kg, estatura média de 1,89 $\pm 0,1 \mathrm{~m}$ ) sem queixas de instabilidade de tornozelo, sem história de lesões agudas dos membros inferiores ou que tivessem requerido afastamento da prática esportiva nos últimos seis meses e sem história de entorses de tornozelo no último ano no membro avaliado. O grupo com instabilidade funcional (GI) foi composto de 21 sujeitos (19 do sexo masculino e dois do feminino, massa média de 80,9 $\pm 12,9 \mathrm{~kg}$, estatura média de 1,91 $\pm 0,1 \mathrm{~m}$ ) com história de pelo menos um entorse que necessitou de afastamento da prática esportiva havia mais de três meses da data da avaliação, queixas de instabilidade - presença de falseios, dificuldade de andar e correr em terrenos irregulares, dificuldade de realizar saltos e mudanças de direção, e reincidência de entorses - e com resultado negativo nos testes de gaveta anterior do tálus, que avalia a integridade do ligamento talofibular anterior ${ }^{(10,21-}$ 22,28-30) e de inclinação talar, que avalia a integridade do ligamento calcaneofibular (21-22,28-30). Essa forma de inclusão também foi utilizada em outros estudos (26,31-38).

Foram avaliados os músculos tibial anterior, gastrocnêmio lateral e fibular longo unilateralmente por meio da eletromiografia de superfície durante um movimento típico do voleibol: aterrissagem após o bloqueio. A aterrissagem após o salto vertical foi escolhida por ser o mecanismo mais comum de entorse de tornozelo(5-6,39-40) e extremamente freqüente na prática do voleibol.

O movimento analisado foi realizado de forma que se aproximasse o máximo possível do fundamento de bloqueio do voleibol. Os atletas que jogam na posição de ponteiro e levantador moveramse lateralmente para o lado do membro avaliado, saltaram com os membros superiores elevados, simulando um bloqueio real, tentaram obter o maior alcance possível e aterrissaram utilizando sua estratégia de aterrissagem normal. Os atletas que jogam na posição de meio de rede realizaram o mesmo movimento, com a diferença de que antes do salto vertical foi realizado o deslocamento lateral cruzado. Essa diferença foi importante a fim de que os atletas realizassem o movimento a que estavam mais adaptados, de acordo com a posição em que jogavam. O movimento de bloqueio foi repetido até que quatro tentativas válidas fossem obtidas. Cada tentativa foi considerada válida quando o indivíduo aterrissava com ambos os pés dentro da área da plataforma de força (figura 1).

Os sujeitos foram avaliados após um período de cinco minutos de aquecimento e habituação à tarefa a ser executada. Para o grupo com instabilidade funcional foi escolhido para análise o tornozelo em que o atleta apresentava queixas de instabilidade, ou em caso de instabilidade bilateral, foi considerado o lado de maior queixa. Para o grupo controle, a escolha foi aleatória.

Utilizou-se para a aquisição do sinal eletromiográfico um eletromiógrafo da EMG System do Brasil (Brasil), que possui oito canais analógicos e eletrodos descartáveis (Medi-Trace 200 series, Kendall-LTP) de $\mathrm{Ag} / \mathrm{AgCl}$, circulares, com 10mm de diâmetro, e um hidrogel condutivo adesivo, colocados de forma que a distância intereletrodo foi de $25 \mathrm{~mm}$ centro a centro. O sinal foi pré-amplificado no local do eletrodo em 20 vezes e enviado ao amplificador do eletromiógrafo (faixa 


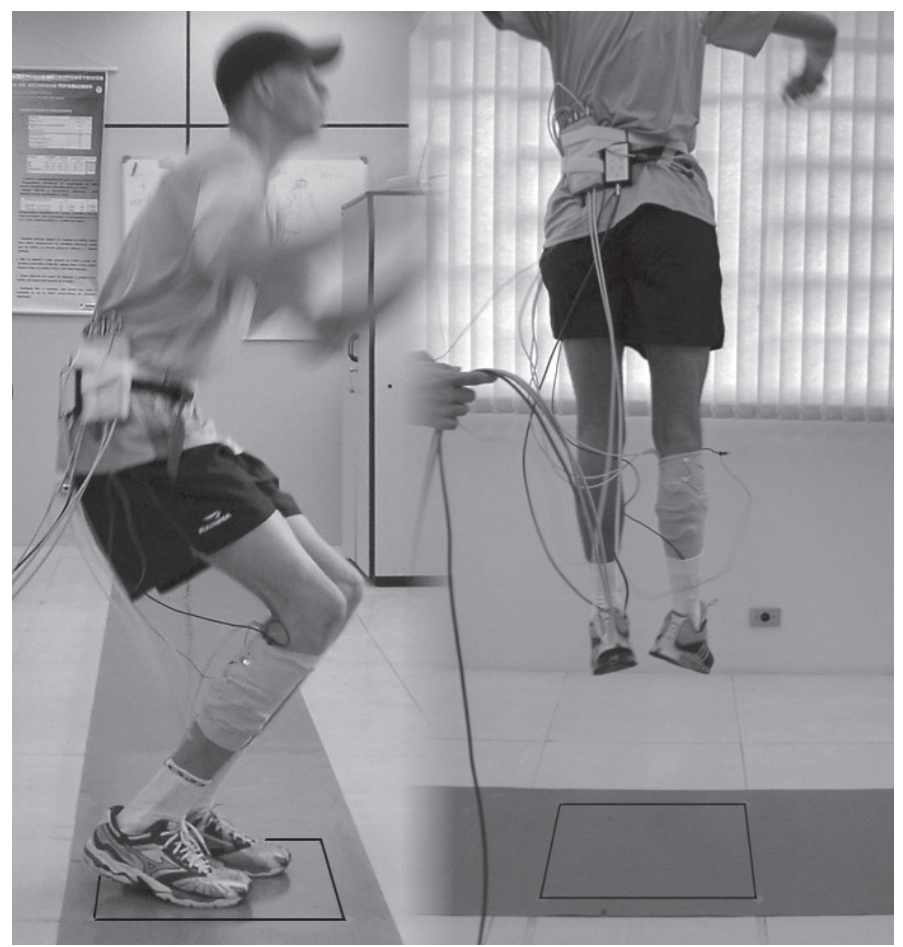

Figura 1. Indivíduo executando o movimento de bloqueio sobre a plataforma de força: realizando a impulsão (à esquerda) e na fase aérea (à direita); o quadrado no solo ilustra a área delimitada pela plataforma de força.

de freqüência: 20 a $500 \mathrm{~Hz}$; taxa de ruído do sinal: 3MV RMS; CMMR: $100 \mathrm{~dB})$, cujo ganho é de fator 50, totalizando ganho de 1.000 para o sinal eletromiográfico.

Para a colocação dos eletrodos foram realizadas a tricotomia e a abrasão da pele com gaze embebida em álcool nos locais de fixação a fim de remover a gordura presente na superfície de contato, diminuindo assim a sua impedância. Os eletrodos foram fixados no ventre muscular, afastados do ponto motor, de forma paralela à da direção das fibras musculares, de acordo com o European concerted action - surface EMG for a non-invasive assesment of muscles (SENIAM) ${ }^{(41-42)}$ e reforçados com fita adesiva do tipo transpore na pele e faixa elástica envolvendoos para evitar artefatos do movimento. O eletrodo de referência foi fixado na tuberosidade da tíbia do membro analisado.

A aquisição da EMG foi sincronizada por meio de um conversor A/D de 12 bits de resolução (placa de aquisição DT3002, AMTI) com a componente vertical da FRS obtida por uma plataforma de força (modelo OR6-7, AMTI), embutida no chão do laboratório e coberta por uma passarela de borracha de $15 \mathrm{~mm}$ de espessura. Os dados da plataforma de força foram amplificados com ganho de 1.000 (sistema MSA-6, AMTI). A componente vertical da FRS permitiu o reconhecimento dos instantes de saída do solo e de impacto após o bloqueio realizado pelos atletas. A freqüência de amostragem de sinais foi de $1.000 \mathrm{~Hz}$ e o tempo de aquisição, de quatro segundos.

\section{TRATAMENTO MATEMÁTICO DOS DADOS}

Os dados foram processados em ambiente de programação MatLab versão 6.5 (Math Works, Inc.). Identificou-se inicialmente o instante da decolagem, em que houve a saída do pé do solo, e o instante do impacto, através da componente vertical da FRS.

A duração da fase aérea, ou o tempo de vôo, foi determinada a fim de verificar se houve diferença entre a altura do salto vertical dos grupos controle e com instabilidade. A altura do salto foi determinada através da equação do movimento de um corpo em queda livre a partir de uma velocidade inicial nula, uma vez que a velocidade do indivíduo na altura máxima atingida é zero:

$$
h(t)=\frac{g t^{2}}{2}
$$

Onde $h$ é a altura alcançada, $g$ corresponde à aceleração da gravidade $\left(9,8 \mathrm{~m} / \mathrm{s}^{2}\right)$ e $t$ é o tempo transcorrido entre a altura máxima atingida e o impacto.

Como o tempo de subida é igual ao tempo de descida, $t$ correspondeu à metade do tempo de vôo do salto vertical.

O sinal eletromiográfico foi analisado em um período correspondente a um ciclo de movimento (figura 2): intervalo entre $200 \mathrm{~ms}$ antes do instante do impacto até 200ms após o instante de impacto, conforme Madigan e Pidcoe ${ }^{(43)}$. O final do ciclo de movimento estudado foi determinado como 200ms após o impacto porque, logo após o choque com o solo, o indivíduo realiza ajustes posturais a fim de estabilizar o centro de gravidade. Como as estratégias de ajustes posturais são muito individuais, procurou-se evitar a abrangência dessa fase de ajuste, a fim de não haver interferência desses eventos no sinal EMG e, portanto, buscar a representação da resposta imediata do indivíduo ao impacto. Esse período compreende a maior parte da absorção do impacto e a desaceleração do corpo.

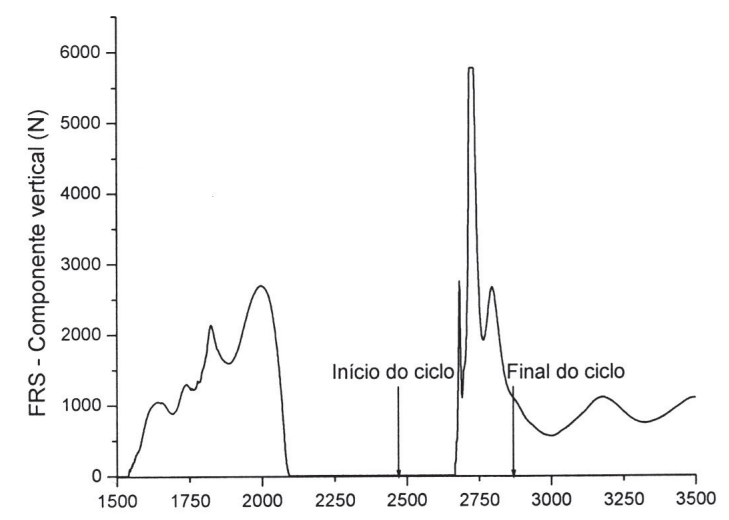

Tempo (ms)

Figura 2. Componente vertical da FRS durante o movimento de bloqueio: a primeira curva observada corresponde ao instante em que o indivíduo realiza o impulso e se prepara para a fase de vôo; o instante de saída do solo correspondeu ao último valor positivo observado antes que o sinal se tornasse nulo (fase aérea); a segunda curva corresponde à aterrissagem; o instante do impacto correspondeu ao primeiro valor positivo observado após o término da fase aérea. As linhas verticais identificam o ciclo do movimento estudado.

Os valores de contração voluntária isométrica máxima (CVIM) foram obtidos a fim de se realizar a normalização dos envoltórios lineares e dos valores das magnitudes dos picos máximos dos músculos estudados $^{(44)}$. Para se obter a CVIM, os músculos foram testados por meio de testes de função, segundo Kendall et al. ${ }^{(45)}$; durante esses testes foram coletados quatro segundos de atividade máxima de cada um dos músculos; a coleta foi repetida duas vezes. Para o cálculo dos 
valores de CVIM, o sinal de aquisição foi retificado por onda completa e filtrado com filtro passa-baixa tipo butterworth de $4^{\mathrm{a}}$ ordem com freqüência de corte de $5 \mathrm{~Hz}$. A partir daí, identificou-se o intervalo de um segundo de maior atividade nas duas aquisições feitas coletadas e calculou-se a média de cada sinal nesse intervalo. Esse valor foi usado para normalizar a atividade muscular.

O padrão temporal de ativação muscular foi obtido pelo cálculo do envoltório linear durante a fase selecionada para a tarefa motora - 200ms antes do impacto, que correspondeu a 0\% do ciclo, a 200ms após o choque, que correspondeu a 100\% do ciclo. Realizou-se, então, uma análise tanto quantitativa quanto qualitativa desses padrões temporais de ativação.

As variáveis quantitativas dos padrões temporais de ativação obtidos pelos envoltórios estão descritas na figura 3, sendo elas: instante do pico máximo e magnitude do pico máximo do tibial anterior (figura 3-1); instante do pico máximo e magnitude do pico máximo do fibular longo (figura 3-2); instante do pico máximo e magnitude do pico máximo do gastrocnêmio lateral (figura 3-3).

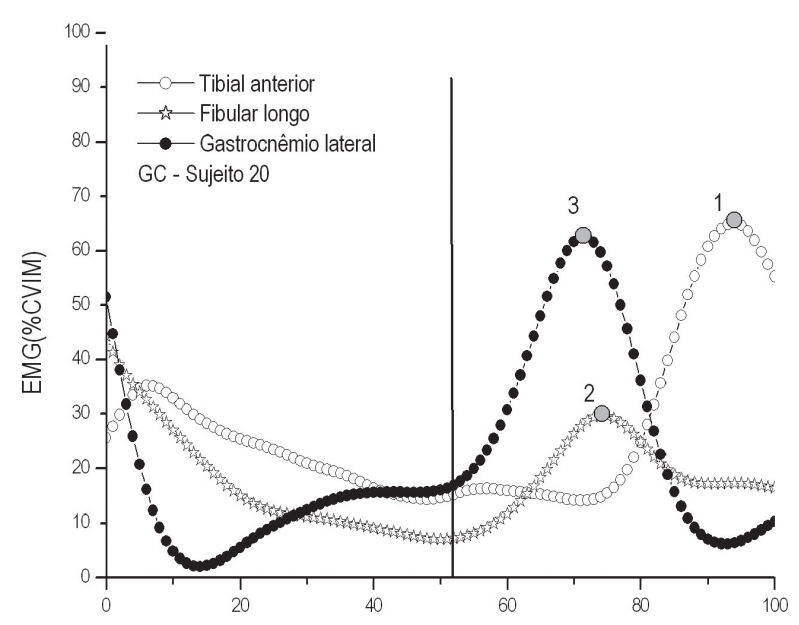

Tempo (\%Ciclo do movimento)

Figura 3. Representação gráfica das variáveis analisadas do envoltório linear dos músculos tibial anterior (TA), fibular longo (FL) e gastrocnêmio lateral (GL) durante o movimento de bloqueio no volei: (1) instante do pico máximo de ativação do TA (eixo x) e pico máximo do TA (eixo y), (2) instante do pico máximo de ativação do FL (eixo x) e pico máximo do TA (eixo y), (3) instante do pico máximo de ativação do GL (eixo x) e pico máximo do TA (eixo y); a linha vertical representa o instante do impacto.

O instante do pico máximo de cada um dos músculos estudados foi referenciado em relação ao instante da aterrissagem no bloqueio. valores positivos correspondem à ocorrência de um pico de ativação anterior ao instante do impacto, e valores negativos, a um pico de ativação após o impacto, identificado pela FRS vertical.

\section{TRATAMENTO ESTATÍSTICO DOS DADOS}

Para a análise estatística, foi utilizado o software SPSS, versão 10.0 (SPSS, Inc.). Foi realizada a análise descritiva das variáveis e testada a normalidade na distribuição das variáveis de razão por meio do teste de Shapiro-Wilks.

As análises inferenciais entre grupos GC e Gl foram feitas utilizando-se o teste $t$ independente, uma vez que as variáveis apresentaram distribuição normal. Foi adotado o valor de $\alpha$ de 0,05 para considerar diferenças significativas.

\section{RESULTADOS}

Não foram encontradas diferenças entre os grupos em relação à altura do salto $(p=0,315)$. O grupo controle apresentou em média ( \pm 1 desvio-padrão) altura de salto de $42,1 \mathrm{~cm}( \pm 7,6)$ e o grupo com instabilidade, $41,0 \mathrm{~cm}( \pm 6,1)$. Os padrões dos envoltórios lineares dos grupos GC e Gl podem ser observados nas figuras 4 e 5, respectivamente.

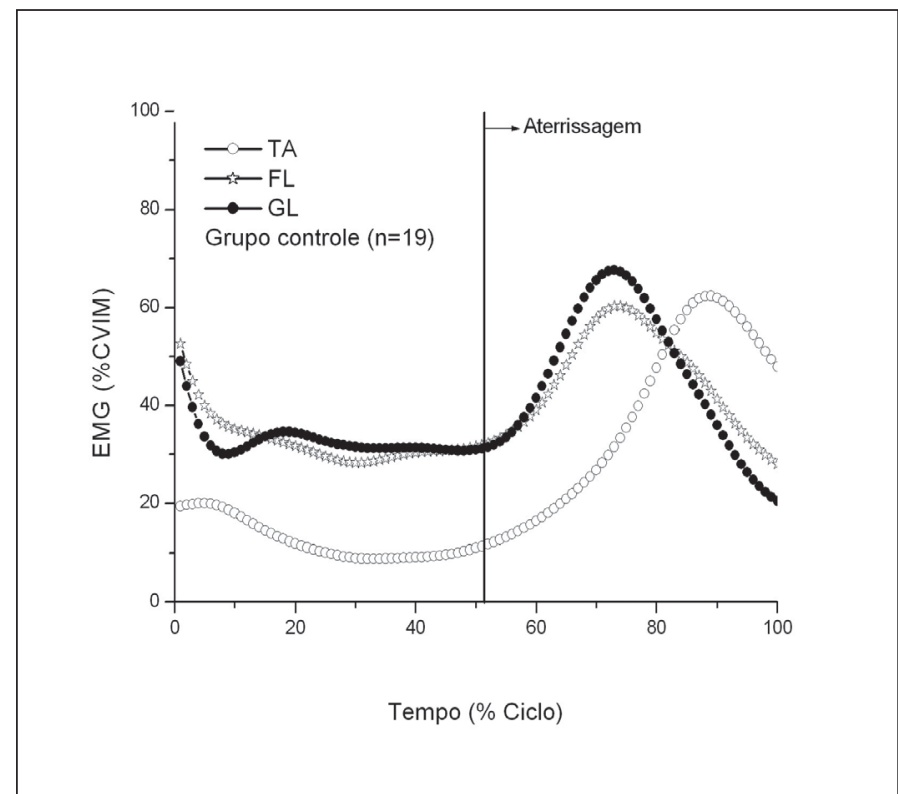

Figura 4. Curvas médias dos envoltórios lineares da EMG dos músculos tibial anterior ( $T A$ ), fibular longo ( $F L)$ e gastrocnêmio lateral $(G L)$ do grupo controle durante a aterrissagem; a linha vertical representa o instante do impacto.

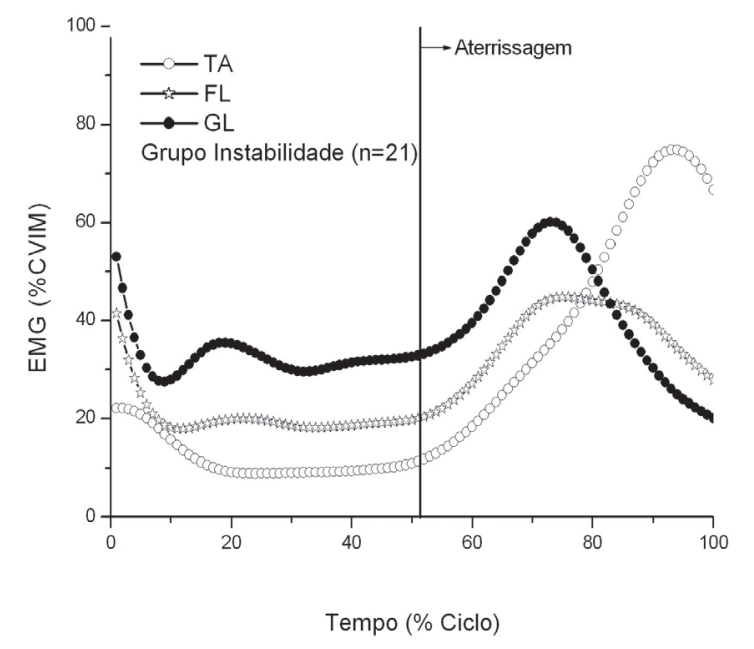

Figura 5. Curvas médias dos envoltórios lineares da EMG dos músculos tibial anterior (TA), fibular longo (FL) e gastrocnêmio lateral (GL) do grupo instabilidade durante a aterrissagem; a linha vertical representa o instante do impacto. 
De forma qualitativa, identifica-se que no grupo controle todos os músculos começam sua atividade antes do instante do impacto e esta aumenta de forma contínua e gradual. Porém, esse aumento é mais acentuado para os músculos $F L$ e GL, uma vez que ambos atingem o máximo de sua atividade a cerca de $75 \%$ do ciclo, o que corresponderia a 100ms após o impacto, enquanto o músculo TA apresenta atividade mais pronunciada mais próxima ao final do ciclo de movimento estudado. No período de tempo que antecede o impacto, a atividade do músculo TA no grupo controle é menor do que a dos músculos FL e do GL.

No grupo com instabilidade o padrão observado é o mesmo em relação ao controle, com a diferença de que antes da aterrissagem há diminuição considerável da diferença entre a atividade dos músculos TA e FL. Além disso, o músculo TA apresenta maior atividade na fase após o impacto nos sujeitos com instabilidade quando se observam os indivíduos do grupo controle.

O músculo GL apresentou seu pico de ativação de forma mais próxima ao instante do impacto para ambos os grupos e semelhante estatisticamente (tabela 1). Os músculos TA e FL apresentaram seus picos de ativação após o instante do impacto, sendo, portanto, representados com sinal negativo (tabela 1), porém se mostraram diferentes entre os grupos. Como não houve diferenças entre os grupos na altura do salto realizado, foi possível representar o inicio da ativação muscular em valores absolutos (ms).

Tabela 1. Diferença de tempo entre o instante da aterrissagem e o instante do pico máximo da atividade eletromioráfica (ms, média \pm 1 desvio-padrão) e valores de $p$ nos grupos controle (GC) e instabilidade (GI)

\begin{tabular}{c|c|c|c}
\hline Músculo & GC (ms) & GI (ms) & p \\
\hline TA & $-107,4 \pm 29,6$ & $-133,98 \pm 25,99^{*}$ & 0,002 \\
\hline$F L$ & $-11,0 \pm 55,9$ & $-41,7 \pm 49,8^{*}$ & 0,037 \\
\hline$G L$ & $4,4 \pm 47,12$ & $-3,5 \pm 39,5$ & 0,358 \\
\hline
\end{tabular}

* Diferença estatisticamente significante

Tanto o músculo TA quanto o FL apresentaram, de forma estatisticamente significante, pico de ativação mais tardio nos sujeitos do grupo Gl (24,8\% mais tardio para TA e 279,1\% mais tardio para FL). 0 músculo TA apresentou pico de maior magnitude (19\% do GC), e o músculo FL, de menor magnitude (-19\% do GC, ambos de forma estatisticamente significante) nos indivíduos com instabilidade funcional do tornozelo (tabela 2). A magnitude do músculo GL foi semelhante nos dois grupos.

Tabela 2. Diferença de magnitude entre os picos de ativação máxima (\%CVIM, média \pm 1 desvio-padrão) e valores de p nos grupos controle (GC) e instabilidade (GI)

\begin{tabular}{c|c|c|c}
\hline Músculo & GC (\%CVIM) & GI (\%CVIM) & p \\
\hline TA & $68,5 \pm 17,2$ & $81,2 \pm 28,8^{*}$ & 0,048 \\
\hline $\mathrm{FL}$ & $72,9 \pm 27,3$ & $59,1 \pm 16,0^{*}$ & 0,032 \\
\hline $\mathrm{GL}$ & $83,3 \pm 32,8$ & $76,6 \pm 26,1$ & 0,239 \\
\hline
\end{tabular}

* Diferença estatisticamente significante

\section{DISCUSSÃO}

O objetivo do presente estudo foi comparar os padrões temporais e de magnitude do tibial anterior, do fibular longo e do gastrocnêmio lateral durante a aterrissagem do salto vertical após o movimento de bloqueio do voleibol entre jogadores com instabilidade funcional de tornozelo e jogadores sem a instabilidade. Os jogadores de voleibol com instabilidade funcional apresentaram menor ativação do fibular longo na fase pré-aterrissagem e ativação atrasada em relação ao instante do impacto, além de maior ativação e pico de ativação mais tardio para o músculo tibial anterior.

Como não houve diferença entre os grupos na altura do salto realizado, nem de performance entre esses indivíduos, pode-se considerar que as diferenças observadas entre os grupos nas variáveis analisadas ocorreram fundamentalmente devido à condição de instabilidade, e não a diferenças de performance entre os grupos.

O movimento analisado foi realizado de forma que se aumentou a variabilidade das respostas eletromiográficas, pois se procurou realizar um movimento mais ecologicamente válido. Porém, cabe ressaltar que o movimento foi analisado em ambiente de laboratório, sem a presença da rede ou da bola de voleibol, não tornando, portanto, a situação exatamente próxima ao do real. Assim, deve-se tomar cuidado ao extrapolar os resultados obtidos neste estudo para a população em geral.

Nos indivíduos do grupo controle, os músculos FL e GL atingiram o máximo de sua atividade cerca de $100 \mathrm{~ms}$ após o impacto, provavelmente aumentando muito os momentos extensores e eversores do complexo tornozelo-pé. No período que antecede o impacto, a atividade do músculo TA é menor do que a atividade dos músculos FL e GL, podendo gerar menores momentos inversores na fase préaterrissagem, momento este que predisporia a maior instabilidade na aterrissagem, aumentando as possibilidades de entorse. Assim, esse padrão de atividade observado antes do impacto levou a aumento da atividade eversora no complexo tornozelo-pé em indivíduos saudáveis, a fim de prepará-lo para o torque inversor gerado pelo contato com o solo após a aterrissagem, e a aumento da atividade do músculo GL a fim de auxiliar na absorção do impacto após a aterrissagem. Esse padrão de ativação do músculo GL também foi descrito por Arampatzis et al. ${ }^{(47)}$ para a aterrissagem a partir de alturas controladas. McKinley e Pedotti ${ }^{(48)}$ sugerem que a extensão do tornozelo na aterrissagem é importante, pois permite que o tornozelo tenha maior movimentação, e, portanto, mais tempo para distribuir as forças de impacto.

No grupo Gl, observou-se diminuição considerável da diferença entre a atividade dos músculos TA e FL, que foi verificada claramente no GC, antes do instante da aterrissagem, o que poderia diminuir a proteção do complexo tornozelo-pé, pois geraria um menor momento eversor no complexo tornozelo-pé. Além disso, observou-se que o músculo FL apresentou pico de ativação menor e mais tardio nos indivíduos com instabilidade, atingindo seu máximo de atividade mais tardiamente no ciclo de movimento estudado. Esse comportamento caracteriza claramente diminuição da função do músculo FL no instante em que o torque inversor é gerado no complexo tornozelo-pé. Assim, esses resultados eletromiográficos predisporiam os indivíduos com instabilidade funcional a maior possibilidade de ocorrer inversão maior do que o suportável pelos estabilizadores estáticos no instante do impacto. 
O músculo TA apresentou atividade mais pronunciada após o instante do impacto, mais próxima ao final do ciclo de movimento estudado em ambos os grupos, da mesma forma que o já descrito na literatura ${ }^{(47-49)}$, e isso leva a maior momento flexor e inversor nos $200 \mathrm{~ms}$ que sucedem o impacto. Arampatzis et al. ${ }^{(47)}$ sugerem que esse aumento da atividade do músculo TA é necessário, pois evitaria eversão excessiva na articulação talocrural. Wilkerson et al.(50) também ressaltaram a função estabilizadora do músculo TA durante atividades em cadeia cinética fechada, tal como na aterrissagem, em que ocorre aplainamento do arco longitudinal do pé. A ação excêntrica dos músculos inversores teria como função controlar a amplitude de eversão do complexo tornozelo-pé, pois se ela for excessiva, ocorre aumento do deslocamento lateral da perna em relação ao pé, que está relativamente estacionário. Esse aumento do deslocamento lateral da perna, e, conseqüentemente, do centro de gravidade, pode fazer com que a borda lateral do pé aja como um fulcro que pode induzir uma inversão súbita do complexo tornozelo-pé. Essa afirmação é reforçada por Munn et al. ${ }^{(51)}$. Assim, o músculo TA teria como função controlar excentricamente essa eversão em cadeia fechada a fim de fornecer estabilidade à articulação. Louwerens et al.(19) também descrevem a função do músculo TA como sendo de absorver o impacto e controlar a extensão e a eversão do complexo tornozelo-pé presentes no início do apoio durante a marcha.

As variáveis quantitativas do envoltório mostraram que o músculo TA apresenta um pico máximo de ativação mais tardio e de maior magnitude nos indivíduos com instabilidade. Se for levada em consideração a função estabilizadora do músculo TA após o impacto, como descrita anteriormente, esse padrão de ativação poderia representar, primeiro, diminuição temporal dessa função estabilizadora do músculo TA, uma vez que seu pico de ativação ocorre de forma mais tardia no ciclo do movimento, e, segundo, resposta adaptativa, representada pelo aumento da magnitude do pico.

Caulfield e Garret ${ }^{(52)}$ avaliaram o salto vertical em indivíduos com IF a partir de uma altura controlada. Eles observaram que os indivíduos com IF mostraram um padrão de maior tendência à posição de flexão de tornozelo no momento da aterrissagem, o que poderia ser conseqüência de maior atividade do músculo TA, que ofereceria maior proteção do complexo ligamentar lateral. Os autores sugerem também que essa seja uma resposta adaptativa à condição de instabilidade, tal como observado no presente estudo.

\section{CONCLUSÃO}

Os jogadores de voleibol com instabilidade funcional de tornozelo apresentaram padrões alterados de ativação nos músculos que estabilizam o complexo tornozelo-pé, em especial no tibial anterior e no fibular longo, em detrimento de um complexo ligamentar aparentemente íntegro, que poderia explicar as queixas apresentadas por esses indivíduos. Esses atletas apresentaram menor ativação do fibular longo na fase pré-aterrissagem e ativação atrasada em relação ao instante do impacto, diminuindo sua capacidade de proteger o complexo tornozelo-pé contra o grande torque inversor gerado no instante do impacto. O tibial anterior apresentou alteração mais marcante e na fase de pós-aterrissagem, mostrando maior ativação e maior pico de atividade. Esse comportamento, por sua vez, parece ser compensatório, uma vez que, após o impacto, a eversão excessiva em cadeia cinética fechada também pode levar à ocorrência de um entorse por inversão.

Portanto, durante a reabilitação, tanto de indivíduos que sofreram um entorse de tornozelo, quanto em indivíduos que apresentam instabilidade funcional, é de grande importância que se dê atenção ao restabelecimento tanto da função quanto do controle da atividade muscular que envolve o complexo tornozelo-pé, em especial, o tibial anterior e o fibular longo, embora pareça um paradoxo, uma vez que esses músculos apresentam funções opostas. Essa recuperação ganha mais importância, uma vez que se observa que realmente os estabilizadores estáticos não são suficientes para dar estabilidade à articulação do tornozelo.

\section{AGRADECIMENTOS}

$$
\text { Apoio Fapesp - processo no 04/14116-1 }
$$

Todos os autores declararam não haver qualquer potencial conflito de interesses referente a este artigo.

\section{REFERÊNCIAS BIBLIOGRÁFICAS}

1. Gross P, Marti B. Risk of degenerative ankle joint disease in volleyball players: study of former elite athletes. Int J Sports Med 1999;20:58-63.

2. Eils E, Rosenbaum D. A multi-station proprioceptive exercise program in patients with ankle instability. Med Sci Sports Exerc 2001;33:1991-8.

3. Schafle MD. Common injuries in volleyball. Treatment, prevention and rehabilitation. Sports Med 1993;16:126-9.

4. Ghirotto FMS, Padovani CR, Gonçalves A. Lesões desportivas: estudo junto aos atletas do XII Campeonato Mundial Masculino de Voleibol. Arq Bras Med 1994;68:307-12.

5. Briner WW Jr, Kacmar L. Common injuries in volleyball. Mechanisms of injury, prevention and rehabilitation. Sports Med 1997;24:65-71.

6. Bahr R, Bahr IA. Incidence of acute volleyball injuries: a prospective cohort study of injury mechanisms and risk factors. Scand J Med Sci Sports 1997;7:166-71.

7. Stasinopoulos D. Comparison of three preventive methods in order to reduce the incidence of ankle inversion sprains among female volleyball players. Br J Sports Med 2004;38:182-5.
8. Dufek JS, Bates BT. Lower extremity performance models for landing. Hum Mov Sci 1992:;299-318.

9. Almeida JMR, Valle LERd, Sacco ICN. Assimetria interlateral da atividade muscular dos membros inferiores no salto vertical. Rev Bras Biomecânica 2001;1:69-78.

10. Nardelli JCC. Estudo epidemiológico de lesões do aparelho locomotor em atletas de voleibol de alto nível. São Paulo: Universidade de São Paulo, 2001.

11. Lynch SA, Renstrom PA. Treatment of acute lateral ankle ligament rupture in the athlete. Conservative versus surgical treatment. Sports Med 1999;27:61-71.

12. Demeritt KM, Shultz SJ, Docherty CL, Gansneder BM, Perrin DH. Chronic ankle instability does not affect lower extremity functional performance. J Athl Train 2002;37:507-11.

13. Osborne MD, Rizzo TD, Jr. Prevention and treatment of ankle sprain in athletes. Sports Med 2003;33:1145-50.

14. Hertel J. Functional instability following lateral ankle sprain. Sports Med 2000;29:361-71.

15. Tropp H. Commentary: Functional Ankle Instability Revisited. J Athl Train 2002;37:512-5. 
16. Monaghan K, Delahunt E, Caulfield B. Ankle function during gait in patients with chronic ankle instability compared to controls. Clin Biomech (Bristol, Avon) 2006;21:168-74.

17. Birmingham TB, Chesworth BM, Hartsell HD, Stevenson AL, Lapenskie GL, Vandervoort AA. Peak passive resistive torque at maximum inversion range of motion in subjects with recurrent ankle inversion sprains. J Orthop Sports Phys Ther 1997;25:342-8.

18. Zampieri C, Almeida GL. Instabilidade funcional do tornozelo: controle motor e aplicação fisioterapêutica. Rev Bras Fisioterapia 2003;7:101-14.

19. Louwerens JW, van Linge B, de Klerk LW, Mulder PG, Snijders CJ. Peroneus longus and tibialis anterio muscle activity in the stance phase. A quantified electromyographic study of 10 controls and 25 patients with chronic ankle instability. Acta Orthop Scand 1995;66:517-23.

20. Gerber JP, Williams GN, Scoville CR, Arciero RA, Taylor DC. Persistent disability associated with ankle sprains: a prospective examination of an athletic population. Foot Ankle Int 1998;19:653-60

21. Safran MR, Benedetti RS, Bartolozzi AR 3rd, Mandelbaum BR. Lateral ankle sprains: a comprehensive review: Part 1: Etiology, pathoanatomy, histopathogenesis, and diagnosis. Med Sci Sports Exerc 1999;31:S429-37.

22. Konradsen $\mathrm{L}$, Olesen $\mathrm{S}$, Hansen HM. Ankle sensorimotor control and eversion strength after acute ankle inversion injuries. Am J Sports Med 1998;26:72-7.

23. Konradsen L, Ravn JB. Prolonged peroneal reaction time in ankle instability. Int J Sports Med 1991;12:290-2.

24. Lofvenberg R, Karrholm J, Sundelin G, Ahlgren O. Prolonged reaction time in patients with chronic lateral instability of the ankle. Am J Sports Med 1995;23:414-7.

25. Sheth P, Yu B, Laskowski ER, An KN. Ankle disk training influences reaction times of selected muscles in a simulated ankle sprain. Am J Sports Med 1997;25:538-43.

26. Ebig M, Lephart SM, Burdett RG, Miller MC, Pincivero DM. The effect of sudden inversion stress on EMG activity of the peroneal and tibialis anterior muscles in the chronically unstable ankle. J Orthop Sports Phys Ther 1997;26:73-7.

27. Gruneberg C, Nieuwenhuijzen PH, Duysens J. Reflex responses in the lower leg following landing impact on an inverting and non-inverting platform. J Physiol 2003;550:985-93.

28. Baumhauer JF, Alosa DM, Renstrom AF, Trevino S, Beynnon B. A prospective study of ankle injury risk factors. Am J Sports Med 1995;23:564-70

29. Jaivin JS, Ferkel RD. Ankle and foot injuries. In: Fu FH, editor. Sports injuries: mechanisms - prevention - treatment. Baltimore: Williams \& Wilkins, 1994;997-1000.

30. Hertel J, Denegar CR, Monroe MM, Stokes WL. Talocrural and subtalar joint instability after latera ankle sprain. Med Sci Sports Exerc 1999;31:1501-8

31. Bernier $\mathrm{JN}$, Perrin DH. Effect of coordination training on proprioception of the functionally unstable ankle. J Orthop Sports Phys Ther 1998;27:264-75

32. Matsusaka N, Yokoyama S, Tsurusaki T, Inokuchi S, Okita M. Effect of ankle disk training combined with tactile stimulation to the leg and foot on functional instability of the ankle. Am J Sports Med 2001;29:25-30.

33. Lentell G, Baas B, Lopez D, McGuire L, Sarrels M, Snyder P. The contributions of proprioceptive deficits, muscle function, and anatomic laxity to functional instability of the ankle. J Orthop Sports Phys Ther 1995;21:206-15.

34. Hubbard TJ, Kaminski TW, Vander Griend RA, Kovaleski JE. Quantitative assessment of mechanical laxity in the functionally unstable ankle. Med Sci Sports Exerc 2004;36:760-6.

35. Vaes P, Duquet W, Handelberg F, Casteleyn PP, Van Tiggelen R, Opdecam P. Objective roentgenologic measurements of the influence of ankle braces on pathologic joint mobility. A comparison of 9 braces. Acta Orthop Belg 1998;64:201-9.

36. Vaes $P$, Van Gheluwe B, Duquet W. Control of acceleration during sudden ankle supination in people with unstable ankles. J Orthop Sports Phys Ther 2001;31:741-52.

37. Santilli V, Frascarelli MA, Paoloni M, Frascarelli F, Camerota F, De Natale L, et al. Peroneus longus muscle activation pattern during gait cycle in athletes affected by functional ankle instability: a surface electromyographic study. Am J Sports Med 2005;33:1183-7.

38. Eils E, Demming C, Kollmeier G, Thorwesten L, Volker K, Rosenbaum D. Comprehensive testing of 10 different ankle braces. Evaluation of passive and rapidly induced stability in subjects with chronic ankle instability. Clin Biomech (Bristol, Avon) 2002;17:526-35.

39. Bahr R, Karlsen R, Lian O, Ovrebo RV. Incidence and mechanisms of acute ankle inversion injuries in volleyball. A retrospective cohort study. Am J Sports Med 1994;22:595-600.

40. Aagaard H, Jorgensen U. Injuries in elite volleyball. Scand J Med Sci Sports 1996;6:228-32.

41. Hermens HJ, Freriks B, Disselhorst-Klug C, Rau G. Development of recommendations for SEMG sensors and sensor placement procedures. J Electromyogr Kinesiol 2000;10:361-74.

42. SENIAM Project, 2005 [cited 200505 jan 2005]; Available from: www.seniam.org

43. Madigan ML, Pidcoe PE. Changes in landing biomechanics during a fatiguing landing activity. J Electromyogr Kinesiol 2003;13:491-8.

44. Burden AM, Trew M, Baltzopoulos V. Normalisation of gait EMGs: a re-examination. J Electromyogr Kinesiol 2003;13:519-32

45. Kendall FP, McCreary EK, Provence PG. Músculos: provas e funções. São Paulo: Manole, 1995

46. Winter DA. The biomechanics \& motor control of human gait: normal, elderly \& pathological. 2nd ed. Waterloo: University of Waterloo Press, 1991.

47. Arampatzis A, Morey-Klapsing G, Bruggemann GP. The effect of falling height on muscle activity and foot motion during landings. J Electromyogr Kinesiol 2003;13:533-44.

48. McKinley P, Pedotti A. Motor strategies in landing from a jump: the role of skill in task execution. Exp Brain Res 1992;90:427-40.

49. Santello M, McDonagh MJ. The control of timing and amplitude of EMG activity in landing movements in humans. Exp Physiol 1998:83:857-74.

50. Wilkerson GB, Pinerola JJ, Caturano RW. Invertor vs. evertor peak torque and power deficiencies associated with lateral ankle ligament injury. J Orthop Sports Phys Ther 1997;26:78-86.

51. Munn J, Beard DJ, Refshauge KM, Lee RY. Eccentric muscle strength in functional ankle instability. Med Sci Sports Exerc 2003;35:245-50.

52. Caulfield B, Garrett M. Changes in ground reaction force during jump landing in subjects with functional instability of the ankle joint. Clin Biomech (Bristol, Avon) 2004;19:617-21. 